PSICOLOGIA

IBEROAMERICANA
Psicología Iberoamericana ISSN: 1405-0943

revista.psicologia@ibero.mx

Universidad Iberoamericana, Ciudad de México México

\title{
Efecto de la creencia en el mundo justo sobre el sexismo ambivalente
}

González-Rivera, Ilse; Díaz-Loving, Rolando

Efecto de la creencia en el mundo justo sobre el sexismo ambivalente

Psicología Iberoamericana, vol. 27, núm. 2, 2019

Universidad Iberoamericana, Ciudad de México, México

Disponible en: http://www.redalyc.org/articulo.oa?id=133962309005 


\title{
Efecto de la creencia en el mundo justo sobre el sexismo ambivalente
}

\author{
Effect of belief in the just world on ambivalent sexism \\ Ilse González-Rivera glez.psi@gmail.com \\ Universidad Nacional Autónoma de México, México \\ http://orcid.org/0000-0002-5114-0500 \\ Rolando Díaz-Loving \\ Universidad Nacional Autónoma de México, México \\ http://orcid.org/0000-0002-4865-480X
}

Psicología Iberoamericana, vol. 27, núm. 2, 2019

Universidad Iberoamericana, Ciudad de México, México

Recepción: 29 Octubre 2019 Aprobación: 07 Enero 2020

Redalyc: http://www.redalyc.org/ articulo.oa?id=133962309005

Financiamiento

Fuente: Consejo Nacional de Ciencia y

Tecnología (CONACYT)

No de contrato: 581389 , CVU 572614

beca 412829

Beneficiario: Ilse González-Rivera
Resumen: El sexismo hostil se refiere a actitudes negativas hacia las mujeres que las definen como menos competentes que los hombres. El sexismo benevolente se refiere a actitudes supuestamente positivas hacia ellas, pero que refuerzan los roles de género tradicionales. En conjunto, ambos componen el sexismo ambivalente. Por su parte, la creencia en el mundo justo es la creencia de que las personas reciben lo que merecen. La creencia en el mundo justo se ha relacionado con el prejuicio, ya que se ha demostrado que las desigualdades sociales se justifican cognitivamente. Con el objetivo de conocer si la creencia en el mundo justo es predictor de las dimensiones del sexismo ambivalente se realizaron análisis de regresión lineal múltiple. Se utilizó el inventario de sexismo ambivalente y el instrumento de creencia en el mundo justo. Participaron 350 personas mexicanas de entre 18 y 27 años. Se trata de un estudio no experimental transversal de un grupo. Los resultados indican que los factores que componen la creencia en el mundo justo son predictores positivos del sexismo ambivalente en sus dos dimensiones: hostil y benevolente, tanto en hombres como en mujeres, ya que incentiva la legitimización del domino masculino y refuerza el acuerdo con el statu quo, por lo que favorece el acuerdo con creencias sexistas. Se discute el efecto de la creencia en el mundo justo como estrategia de enfrentamiento ante la inequidad y su efecto en el mantenimiento de los prejuicios como el sexismo.

Palabras clave: desigualdad social, justificación de desigualdad, roles de género, sexismo hostil, sexismo benevolente.

Abstract: Hostile sexism refers to negative attitudes towards women that define them as less competent than men. Benevolent sexism refers to supposedly positive attitudes toward woman that reinforce traditional gender roles. The belief in a just world is the belief that people receive what they deserve. This belief is useful to reduce cognitive dissonance by confronting the unequal social system. Prejudice has been related to belief in a just world because this belief allows social inequalities to be justified cognitively. Multiple linear regression analyses were performed to discover whether the belief in a just world was a predictor of sexism and its dimensions. The inventory of ambivalent sexism and the instrument of belief in a just world was used. 350 Mexican people between the ages of 18 and 27-years-old participated. The results indicate that the belief in a just world is a significant predictor of ambivalent sexism in both men and women since it encourages the legitimization of the male domination and reinforces the agreement with the status quo, which favors sexist beliefs. The effect of the belief in a just world as a strategy for confronting inequality and its effects on the maintenance of prejudices is discussed.

Keywords: benevolent sexism, social inequality. 


\section{Introducción}

Los prejuicios son actitudes que implican evaluaciones sobre una persona o grupo de personas por el hecho de pertenecer a cierta categoría social (Brown, 2010; Eagly \& Carli, 2003). Una de las formas más generalizadas del prejuicio es el que se tiene por razones de género. A este prejuicio se le conoce como sexismo.

Los estudios de Glick y Fiske (1996) sobre sexismo proponen que existen dos tipos de sexismo: el sexismo hostil y el sexismo benevolente; ambos componen el sexismo ambivalente. El sexismo hostil se refiere a actitudes explícitamente negativas hacia las mujeres que las definen como incapaces de ejercer poder sobre lo económico, legal y político. El sexismo benevolente se refiere a actitudes hacia las mujeres que son supuestamente positivas, pero que enfatizan los roles de género tradicionales. Este tipo de sexismo parece una actitud positiva hacia las mujeres, pero en realidad refuerza los estereotipos pues provee una racionalización para confirmar el rol de las mujeres dentro del campo doméstico (Cárdenas, Lay, González, Calderón, \& Alegría, 2010).

Es decir, el sexismo incluye no sólo actitudes hostiles y de rechazo hacia las mujeres, sino también actitudes benevolentes que promueven y respaldan los roles de género y la inequidad entre los sexos (Cárdenas et al., 2010). Las dos dimensiones son ideologías que se encuentran a través de diferentes culturas (Glick et al., 2000).

En México se han realizado diversos estudios sobre el sexismo desde la aproximación teórica del sexismo ambivalente, por ejemplo Luna $(2008,2010)$ realizó la validación del instrumento original propuesto por Glick y Fiske (1996), y brinda una aportación teórica de la conceptualización y operacionalización del sexismo en México. Por su parte, Cruz, Zempoaltecatl y Correa (2005), realizan un estudio sobre los perfiles del sexismo en la Ciudad de México encontrando que el sexismo hacia las mujeres implica un dominio social que privilegia a los hombres y que la educación es un aspecto relevante que se debe considerar para combatir el sexismo. Asimismo, Díaz-Loving, González-Rivera y BaezaRivera (2019) realizaron un estudio sobre sexismo en México con una configuración a partir de las premisas histórico-psicosocioculturales.

También se han realizado algunos estudios sobre sexismo hacia los hombres en los que se muestra que este tipo de sexismo es complementario al de las mujeres ya que es parte de una dinámica de poder que aumenta la desigualdad social y que a través de su estudio se comprenden las dinámicas subyacentes en la discriminación hacia ambos sexos (ArenasRojas \& Rojas-Solís, 2015; Gonzalez-Rivera \& Díaz-Loving, 2018). Adicionalmente, Carrera (2018) analiza el uso de metáforas en la construcción del discurso sexista en tres categorías de textos en México, en los que se destacan diferentes retos que las mujeres han tenido y siguen teniendo en el país para obtener un reparto equitativo de las tareas domésticas y de la esfera pública.

De igual forma, estudios en México y en el mundo muestran que el sexismo tiene consecuencias negativas en diferentes ámbitos de la vida de 
las mujeres y de la sociedad en conjunto. Por ejemplo, este prejuicio se ha relacionado con baja escolaridad en las mujeres (Castillo \& Maldonado, 1996; Luna, 2008) con bajo nivel de desarrollo humano del país (Moya, Vasco, Glick, \& Poeschl, 2002), así como con el embarazo adolescente (Acuña \& Montecino, 2014; Cusick, 1987; Fernández, 2018) y con efectos negativos en la salud física y psicológica de las mujeres (Molix, 2014).

Además estudios en diferentes culturas muestran que a las mujeres se les asigna un estatus bajo en la sociedad y en la familia (Chen, Fiske, \& Lee, 2009), lo que contribuye a la constitución del statu quo desigual, de ahí la importancia de realizar investigaciones sobre ello. El estatus atribuido a los grupos sociales se asocia a las expectativas de desempeño y funciona para determinar su valía social. Así, las mujeres como grupo tienen un bajo estatus en las sociedades, mientras que los hombres como grupo se clasifican como pertenecientes a una categoría de alto estatus (Crocker \& Quimm, 2001; Phinney, 1990; Rudman \& Glick, 2001).

Una de las variables con las que se ha relacionado el prejuicio y el respaldo al statu quo es la creencia en el mundo justo. Esta variable se refiere al grado en el que se percibe al entorno y a las normas sociales como apropiadas y legítimas. Es decir, es la creencia de que los individuos pueden tener en mayor o menor medida de que el mundo es un lugar justo y predecible en el que las personas obtienen lo que merecen y merecen lo que reciben (Hafer \& Choma, 2009; Uhlmann, Poehlman, \& Bargh, 2009).

Así, la creencia en el mundo justo es entendida como un recurso que permite enfrentarse constantemente a actos injustos o impredecibles (Cruz, 2013), ya que es útil para reducir la disonancia cognoscitiva, la ansiedad y la incertidumbre, lo que proporciona un sentido de orden y justicia en el mundo (Jost \& Hunyady, 2002, 2005).

Por ejemplo, el acuerdo con la premisa de que cada uno recibe lo que se ha ganado, implica la creencia de que quienes son tratados de manera desigual es porque no han conseguido los méritos necesarios para cambiar su situación o bien, porque tienen características intrínsecas que les brindan el derecho de acceder sólo a ciertos recursos.

De este modo, la creencia en el mundo justo juega un papel fundamental en el mantenimiento de los estereotipos y del prejuicio para los individuos. Esta creencia permite que se justifique cognitivamente las desigualdades sociales experimentadas o presenciadas, brindando un sentido de orden y tranquilidad a las personas.

La creencia en el mundo justo se compone de cuatro factores. La justicia final que se refiere a la idea de que todas las personas reciben tarde o temprano los premios o castigos que merecen de acuerdo al mérito de sus acciones. La derogación que es la reinterpretación de las causas de la injusticia, al señalar que las personas que sufren alguna injusticia son culpables de la misma, ya sea por sus características personales o por negligencia. La distancia que se refiere a la creencia de que las injusticias ocurren a personas diferentes o que viven lejos y que incluso en condiciones de adversidad (pobreza) se puede ser feliz. Y la negación o el 
alejamiento de estímulos que amenazan la creencia en el mundo justo. En conjunto, estos factores tienen una función paliativa, es decir, constituyen una forma de afrontamiento psicológico para sobrellevar las amenazas de un sistema injusto o inestable socialmente (Cruz, 2013; Jost \& Banaji, 1994).

Entonces, la creencia en el mundo justo puede implicar el acuerdo con que los hombres tengan acceso a ciertos recursos (empleo mejor remunerado, puestos de liderazgo, cargos políticos, etcétera), pero las mujeres no, ya que pertenecen a dos categorías sociales que se consideran diferentes. Esta distribución de recursos se percibe como legitima y justa aunque objetivamente implique una distribución desigual entre los miembros de las categorías sociales (Mummendey \& Otten, 2001). También esta creencia implica la aceptación de la propia situación en el estatus social, incluso si se pertenece a un grupo de bajo estatus, puesto que si una persona considera que ha recibido lo que merece, es más probable que acepte las desventajas que implican pertenecer a un grupo de menor estatus en la jerarquía social y que no muestre inconformidad ante ellas (Ellemers \& Barreto, 2001).

En concordancia con lo anterior, existe evidencia experimental que muestra que altos niveles de acuerdo con la creencia en el mundo justo implican percibir la discriminación como algo menos personal, lo que aumenta la probabilidad de justificar el sistema social (Uhlmann et al., 2009). De hecho, en mujeres trabajadoras la creencia en el mundo justo se ha relacionado con menores comportamientos dirigidos a mejorar la propia situación laboral y con mayores creencias pesimistas sobre tales intentos (Hafer \& Choma, 2009; Hafer \& Olson, 1993).

Puesto que de manera histórica y sistemática a las mujeres se les ha considerado como miembros de un grupo de bajo estatus (Ellemers \& Barreto, 2001), se les atribuyen estereotipos que legitiman el dominio social masculino (Cikara, Lee, Fiske, \& Glick, 2009). De hecho, algunos estudios muestran que los hombres tienen la percepción de merecer más en comparación con las mujeres (Blanton, George, \& Crocker, 2001; Crosby, Stockdale, \& Ropp, 1947; Jost, 1997; O’Brien \& Major, 2009; Pelham \& Hetts, 2001).

$\mathrm{Al}$ realizar estudios sobre los fenómenos psicológicos no se puede dejar de lado el contexto social, como el hecho de que en México los hombres tienden a percibirse a sí mismos con atributos instrumentales, como ser dominantes y capaces, pero las mujeres se perciben a sí mismas con atributos expresivos como ser dependientes y cariñosas (Díaz-Loving, Velasco-Matus, \& Rivera-Aragón, 2018; Rocha \& Díaz-Loving, 2004). Aunque esto se ha modificado a lo largo del tiempo, aún prevalecen un fuerte acuerdo respecto al papel de las mujeres en la sociedad mexicana, en la que se les encasilla dentro de los roles de lo doméstico y familiar (Díaz-Loving et al., 2018), por lo que tienen menos estatus, poder y acceso a recursos. Lo cierto es que siguen existiendo obstáculos para ellas en la sociedad y se mantienen premisas culturales que reafirman la desigualdad. Incluso se ha observado que existe una transformación del 
sexismo tradicional en México, pero aún prevalecen elementos asociados a la supremacía de los hombres sobre las mujeres (Díaz-Loving et al., 2019).

De hecho, en un estudio realizado en el norte, centro y sur de México en lugares urbanos, semi-urbanos y rurales, con una muestra por cuotas con educación básica, con nivel medio (técnico y bachillerato) y con educación superior (licenciatura y posgrado) se muestra que aun coexisten normas y creencias tradicionales junto con las no tradicionales; por ejemplo, se muestra acuerdo con la equidad, pero también con el machismo y la virginidad, asimismo, se presentan creencias de emancipación al mismo tiempo que se muestra acuerdo con el sexismo y con la premisa del marianismo. También prevalecen las normas asociadas con el statu quo de los padres y la abnegación de las mujeres (Díaz-Loving et al., 2015). Por lo tanto, a pesar de la creciente ola de investigaciones sobre el empoderamiento femenino que muestran un cambio en los roles de las mujeres, el cambio se esta dando de manera parcial y gradual por lo que aún prevalecen normas y creencias asociadas con la cultura tradicional en los mexicanos.

Asimismo, en algunos estudios se ha mostrado que el sexismo es un elemento presente en diferentes culturas de manera histórica (Acuña \& Montecino, 2014; Verdú \& Briones, 2016). Por ejemplo, Rojas y Moreno (2016) realizaron una investigación sobre el sexismo ambivalente en personas con diversidad étnico-cultural, demostrando que el sexismo varía en función de las características culturales de los individuos, pero se presenta de manera constante en sus creencias. Asimismo, en un estudio realizado en España, Latinoamérica y África se muestran las diferencias en sexismo en función del origen cultural (Arnoso, Babe, Arnoso, \& Elgorriaga, 2017).

El sistema jerárquico de la sociedad mexicana conlleva altos niveles de desigualdad entre hombres y mujeres, implica la distribución desigual de recursos y diversas desventajas entre los grupos. Esto representa un obstáculo para lograr la equidad e implica menor calidad de vida de las mujeres como individuos y como grupo, así como repercusiones en la sociedad mexicana en general. De ahí la importancia de realizar estudios que permitan conocer las variables que se relacionan con el sexismo y contribuir a la construcción de paradigmas que permitan comprender cómo combatir este prejuicio y, de este modo, generar estrategias que sean útiles para transformar el orden social desigual.

Así, la evidencia indica que la creencia en el mundo justo tiene una relación con el prejuicio y con el mantenimiento de un statu quo desigual, por lo que en la presente investigación se plantea que esta variable puede tener un efecto en el sexismo, ya que implica acuerdo con estereotipos y aceptación de situaciones desiguales o injustas para legitimar el sistema jerárquico en el que las mujeres están en desventaja. De ahí que el objetivo de la presente investigación sea conocer el efecto de la creencia en el mundo justo sobre el sexismo ambivalente en una muestra de mujeres y hombres mexicanos. Para responder a esta pregunta, se parte de la aproximación teórica del sexismo ambivalente propuesta por Glick y Fiske (1996). 
La principal hipótesis que sustenta la investigación es que los diferentes factores de la creencia en el mundo justo tendrán un efecto positivo sobre los factores del sexismo tanto en su dimensión benevolente como en su dimensión hostil. Tanto hombres como mujeres utilizarían la creencia en el mundo justo para enfrentarse al sistema social: en el caso de las mujeres fungiría como un mecanismo paliativo, mientras que en los hombres como un mecanismo que justifica la distribución de recursos a su favor.

\section{Método}

\section{Diseño}

Se trata de un estudio no experimental transeccional (transversal) de un grupo. El alcance de la investigación es correlacional.

\section{Participantes}

Se realizó un muestreo no probabilístico intencional con participación voluntaria y anónima. Participaron 350 personas, 200 mujeres y 150 hombres de entre 18 y 27 años $(x \#=20.98$, de=2.27), $72.6 \%$ con escolaridad licenciatura, $19.4 \%$ con escolaridad preparatoria, $7.7 \%$ escolaridad básica y $3 \%$ posgrado. Del total de la muestra $74 \%$ se dedicaban a estudiar y $93 \%$ eran solteros.

\section{Instrumentos}

Inventario de sexismo ambivalente (Glick \& Fiske, 1996)

El inventario de sexismo ambivalente fue adaptado y validado en México encontrando un índice de confiabilidad mediante la prueba alfa de Chronbach de .85 y de .92 (Luna, 2008, 2010). Se compone de 24 reactivos en formato tipo Likert con siete opciones de respuesta donde 1 corresponde a nada cierto y 7 a muy cierto. La dimensión de sexismo hostil contiene reactivos como las mujeres intentan ganar poder controlando a los hombres; mientras que la dimensión de sexismo benevolente contiene reactivos como las mujeres deben ser queridas y protegidas por los hombres. La configuración factorial encontrada para la muestra del presente estudio tiene un alfa de Cronbach total de .87 y se compone de un factor de sexismo hostil con un alfa de Cronbach de .84, y dos factores de sexismo benevolente: intimidad heterosexual con un alfa de Cronbach de .79 y diferenciación complementaria de género con un alfa de Cronbach de.71. 


\section{Instrumento de creencia en el mundo justo}

Creado y validado para población mexicana (Cruz, 2013) con una estructura factorial que explica el $62.3 \%$ de la varianza y un índice de confiabilidad mediante la prueba alfa de Chronbach de .84. Se compone de 17 reactivos que se presentan en formato tipo Likert de 7 opciones de respuesta donde 1 corresponde a nada cierto y 7 a muy cierto. La escala se compone de 4 factores: justicia final con un alfa de Cronbach de .85; derogación con un alfa de Cronbach de .72; distancia con un alfa de Cronbach de .70; y negación con un alfa de Cronbach de .76. Contiene reactivos como: al final, las personas siempre reciben lo que se merecen; quienes pasan muchas injusticias en su vida es porque no han tenido el valor para defenderse.

\section{Cuestionario sociodemográfico}

Se recolectó información sobre las características personales de los participantes con un cuestionario de datos sociodemográficos, solicitando los siguientes datos: estado civil, edad, nivel educativo y ocupación.

\section{Procedimiento}

La aplicación se llevó a cabo de forma individual a población abierta en espacios públicos como plazas, parques, y escuelas, siguiendo un muestreo no probabilístico. Los cuestionarios fueron aplicados por psicólogos previamente capacitados. Se siguieron los lineamientos éticos indicados en el Código Ético del Psicólogo. Al momento de la aplicación se entregó un consentimiento informado que los participantes leyeron y firmaron antes de comenzar en donde se explicó de manera general el objetivo de investigación, se garantizó la confidencialidad y anonimato de los datos, así como el uso exclusivo de ellos con fines de investigación. La participación fue voluntaria. Posteriormente, se les explicaron las instrucciones a seguir, y se entregó a los participantes un cuadernillo tamaño carta que contenía las instrucciones para contestar los instrumentos, el inventario de sexismo ambivalente, el instrumento de creencia en el mundo justo y el cuestionario de datos sociodemográficos. Cuando terminaron de contestar se les agradeció su participación.

Una vez recolectada la información se capturó en una base de datos y posteriormente se siguieron los procedimientos propuestos por Reyes Lagunes y García y Barragán (2008) por escala. Este procedimiento consiste en lo siguiente: análisis de frecuencias de cada reactivo incluyendo sesgo; determinar la capacidad discriminativa de cada reactivo; obtener indicadores de consistencia interna mediante alfa de Cronbach; análisis de componentes principales; rotación ortogonal (tipo Varimax); y normalización de Kaiser para obtener evidencias de la validez de constructo del instrumento. 
Con este procedimiento se confirmó que las escalas utilizadas eran válidas y confiables para la muestra aplicada con el objetivo de confirmar la estructura factorial de los reactivos dadas las características de la muestra, de este modo se verifica que sean culturalmente sensibles a la situación y contexto de los participantes. Posteriormente se realizaron análisis de regresión múltiple utilizando los factores de la creencia en el mundo justo como predictores del sexismo ambivalente. Todos los datos se analizaron en el paquete estadístico IBM SPSS (Statistical Product and Service Solutions) en su versión 21.00.

\section{Resultados}

Para garantizar la validez del modelo, se verificó que los datos cumplieran con los supuestos para realizar un modelo de regresión lineal. Los datos cumplieron con los supuestos: linealidad, independencia y normalidad. El supuesto de linealidad se obtuvo a través de la obtención de los diagramas de regresión parcial; el supuesto de independencia se obtuvo a través del estadístico de Durbin-Watson $(\mathrm{dw}=1.9)$; y el supuesto de normalidad se verificó a través del un histograma de residuos tipificados. Posteriormente, se encontró un modelo predictor del sexismo hostil y del sexismo benevolente mediante el procedimiento de regresión múltiple con el método enter. A continuación, se presentan los resultados por cada factor.

La dimensión de sexismo hostil se compone de un factor, mientras que la dimensión de sexismo benevolente se compone de dos factores: intimidad heterosexual, y diferenciación complementaria de género. La Tabla 1 muestra que la justicia final y la derogación son factores de la creencia en el mundo justo que resultaron predictores significativos del sexismo hostil, mientras que la distancia y la negación no fueron factores significativos para predecir este tipo de sexismo. 
Tabla 1

Análisis de regresión lineal múltiple: creencia en el mundo justo como predictor del sexismo hostil

Tabla 1

Análisis de regresión lineal múltiple: creencia en el mundo justo como predictor del sexismo hostil

\begin{tabular}{lll}
\hline Predictor: & Sexismo hostil & $\mathrm{t}$ \\
Creencia en el mundo justo & & \\
\hline Justicia final & $.27^{\star \star}$ & $3.2^{\star \star \star}$ \\
Derogación & $.32^{\star \star \star}$ & $3.6^{\star \star \star}$ \\
Distancia & $.23^{\star \star}$ & 1.55 \\
Negación & $.19^{\star \star}$ & 1.35 \\
& & \\
$R^{2}$ & .38 & \\
$F(4,369)$ & $15.8^{\star \star \star}$ & \\
\hline
\end{tabular}

Nota. ${ }^{\star} p<.05 .{ }^{\star \star} p<.01 .{ }^{\star \star *} p<.001$.

$$
\text { Nota: }{ }^{*} \mathrm{p}<.05 .{ }^{* *} \mathrm{p}<.01 .{ }^{* * *} \mathrm{p}<.001 .
$$

La Tabla 2 muestra que la justicia final, la distancia, y la negación resultan predictores significativos del sexismo benevolente en su dimensión de intimidad heterosexual, mientras que la derogación no resultó un predictor significativo.

\section{Tabla 2}

Análisis de regresión lineal múltiple: creencia en el mundo justo como predictor del sexismo benevolente intimidad heterosexual

Tabla 2

Análisis de regresión lineal múltiple: creencia en el mundo justo como predictor del sexismo benevolente intimidad heterosexual

\section{Predictor:}

Creencia en el mundo justo

\begin{tabular}{lll}
\hline Justicia final & $.31^{\star \star \star}$ & $4.1^{\star \star \star}$ \\
Derogación & $.29^{\star \star \star}$ & 1.5 \\
Distancia & $.35^{\star \star \star}$ & $4.4^{\star \star \star}$ \\
Negación & $.23^{\star \star \star}$ & $1.9^{\star}$ \\
& & \\
$R^{2}$ & .44 & \\
$F(4,369)$ & $22^{\star \star \star}$ & \\
\hline Nota. ${ }^{\star} p<.05 .{ }^{\star \star} p<.01 .^{\star \star \star} p<.001$.
\end{tabular}

\section{Intimidad heterosexual $t$}

Nota. ${ }^{*} \mathrm{p}<.05 .{ }^{* *} \mathrm{p}<.01 .{ }^{* * *} \mathrm{p}<.001$. 
Tabla 3

Análisis de regresión lineal múltiple: creencia en el mundo justo como predictor del sexismo benevolente diferenciación complementaria de género

Tabla 3

Análisis de regresión lineal múltiple: creencia en el mundo justo como predictor del sexismo benevolente diferenciación complementaria de género

\begin{tabular}{lll}
\hline & $\begin{array}{l}\text { Diferenciación complementaria } \\
\text { Predictor: }\end{array}$ & \multicolumn{1}{l}{$\mathrm{t}$} \\
Creencia en el mundo justo & & $3.22^{\star \star \star}$ \\
\hline Justicia Final & $.27^{\star \star \star}$ & .88 \\
Derogación & $.26^{\star \star \star}$ & $5.0^{\star \star \star}$ \\
Distancia & $.37^{\star \star \star}$ & $2.8^{\star \star}$ \\
Negación & $.26^{\star \star \star}$ & \\
$R^{2}$ & & \\
$F(4,369)$ & .44 & \\
\hline Nota. ${ }^{*} p<.05 .{ }^{\star \star} p<.01 .{ }^{\star \star \star} p<.001$. &
\end{tabular}

Nota. ${ }^{*} \mathrm{p}<.05 .{ }^{* *} \mathrm{p}<.01 .^{* * *} \mathrm{p}<.001$

Finalmente, la Tabla 3 muestra que, para el factor del sexismo benevolente de diferenciación complementaria de género, el factor de justicia final, la distancia y la negación resultaron predictores significativos, mientras que la derogación no mostró ser un factor significativo.

\section{Discusión}

Los resultados obtenidos apoyan las hipótesis planteadas al inicio de la investigación: los factores que componen la creencia en el mundo justo son predictores positivos del sexismo ambivalente en sus dos dimensiones: hostil y benevolente.

El factor de justicia final implica la creencia de que todas las personas reciben tarde o temprano los premios o castigos que merecen de acuerdo con el mérito de sus acciones (Cruz, 2013). Este factor fue un predictor significativo para las dos dimensiones del sexismo ambivalente, tanto de la dimensión hostil como de la benevolente: intimidad heterosexual y diferenciación complementaria de género.

Este resultado indica que, tal como se esperaba, los miembros de grupos de bajo estatus utilizan la creencia en el mundo justo para legitimar sus desventajas sosteniendo que merecen estar en una posición de desventaja (el caso de las mujeres). Mientras que los miembros de grupos de alto estatus, justifican su posición de mayor poder al considerar que lo merecen (para el caso de los hombres). Así, la creencia en el mundo justo funge como una estrategia de enfrentamiento ante la inequidad y contribuye al mantenimiento de los prejuicios.

Por su parte, la derogación fue un factor significativo únicamente como predictor del sexismo hostil. Esto tiene sentido considerando que el sexismo hostil implica actitudes con carga totalmente negativa hacia las mujeres, por lo que es predicho por la creencia de que si las mujeres tienen una posición de desventaja o injusticia social es porque tienen menores 
capacidades que los hombres o porque son intrínsecamente diferentes. Como la derogación en sí misma implica cierto grado de hostilidad, ésta se traduce en hostilidad hacia las mujeres y por lo tanto no resulta significativo como predictor del sexismo benevolente, que tiene un tono considerado positivo (Glick \& Fiske, 1996).

Tanto el factor de distancia como el factor de negación resultaron significativos únicamente para los factores del sexismo benevolente: diferenciación complementaria de género e intimidad heterosexual. La distancia implica la creencia de que las injusticias ocurren a personas diferentes y que incluso en condiciones de adversidad (pobreza, exclusión, discriminación) se puede ser feliz; mientras que la negación implica el alejamiento de estímulos que amenazan la creencia en el mundo justo. Ambas creencias predicen el sexismo benevolente, ya que implica que las mujeres complementan a los hombres y, a su vez, los hombres cuidan a las mujeres por lo que la negación y la distancia robustecen la función compensatoria de este tipo de sexismo (Glick \& Fiske, 1996).

El hecho de que para el sexismo benevolente resultaran predictores significativos la negación y la distancia, mientras que para el sexismo hostil sólo resultara predictor significativo la derogación, indica que, tal como se ha supuesto teóricamente, el sexismo hostil es una forma de castigo para las mujeres, pues implica que ellas merecen tener desventajas debido a que son menos competentes. Mientras que el sexismo benevolente tiene una función compensatoria que justifica el trato desigual bajo la premisa de que son más débiles y con características complementarias a los hombres y, por lo tanto, deben ser protegidas y mantenerse en el campo de los doméstico (Durante, Fiske, Kervyn, Cuddy, Akande, Adetoun, \& Storari, 2013; Moya et al., 2002).

En el presente estudio no se miden variables culturales que permitan comprobar el impacto de la cultura en la creencia en el mundo justo y el sexismo; sin embargo, sí es posible considerar las características culturales de la sociedad mexicana al discutir los resultados encontrados (Arnoso et al., 2017; Díaz-Loving et al., 2015). Si se analizan los resultados tomando en cuenta el contexto cultural de los participantes, se puede notar el papel de la cultura en la que se desarrollan: una cultura colectivista, apegada a las normas, tradicionalista, y que enfatiza los roles de género (Díaz-Guerrero, 1994; Díaz-Loving et al., 2015). Así, la cultura mexicana promueve la necesidad de desarrollar estrategias cognitivas para enfrentarse a la desigualdad, tal como la creencia en el mundo justo, que resulta útil para adaptarse socialmente y aumentar el bienestar subjetivo al percibir el mundo como controlable (Leite, Cardoso, Marques, \& Morais, 2017). Futuras investigaciones deberán explorar las diferencias culturales implicadas en este fenómeno.

El sesgo perceptivo de la creencia en el mundo justo promueve que los individuos tiendan a percibir que los hombres merecen tener un estatus mayor al de las mujeres y que, incluso, cuando se encuentran en la situación de sexismo, tiendan a justificarlo, minimizarlo y ratificar su creencia en los estereotipos de que las mujeres son menos competentes que los hombres. Asimismo, esta creencia es una estrategia que se incentiva en 
los sistemas donde hay altos niveles de desigualdad, como lo es México, para mantener la legitimización del dominio de los grupos de alto estatus y mantener en inacción a los grupos de bajo estatus como las mujeres (Cikara et al., 2009). Las mujeres al ser oprimidas crónicamente a nivel individual e institucional pueden cooperar con el mantenimiento de la inequidad, debido al acuerdo con la creencia en el mundo justo ya que les es útil para evitar el desagrado y la disonancia que se produce al enfrentarse a situaciones de injusticia (Cruz, 2013) tales como los efectos producidos por el sexismo.

El mismo fenómeno ocurre en otros sistemas de inequidad como el económico o sistemas donde existe alta discriminación debida a la condición racial (Kay \& Jost, 2003; Kay \& Zanna, 2009).

En este sentido, la creencia en el mundo justo no sólo implica el mantenimiento de prejuicios como el sexismo, sino también el mantenimiento de estereotipos que refuerzan la desigualdad de clases, de razas y la inacción social (Gatica, Martini, Dreizik, \& Imhoff, 2017; Hafer \& Choma, 2009; Hafer \& Olson, 1993). Así que a pesar de tener una función que se ha planteado como adaptativa, también implica una desventaja para los grupos de estatus bajo y para mantener y legitimar el orden social desigual.

Por lo tanto, es necesario trabajar en estrategias adaptativas que permitan a los individuos enfrentarse al sistema social cambiante, impredecible o injusto, pero que no impliquen el mantenimiento de un statu quo desigual. También es necesario crear estrategias que permitan que los grupos sociales tomen posturas a favor de la igualdad, para reducir la discriminación y el prejuicio hacia las mujeres (Kaiser, Hagiwara, Malahy, \& Wilkins, 2009). Ésta es una tarea que se mantiene vigente para ser abordada en futuras investigaciones que permitan no sólo identificar el comportamiento de las variables como la creencia en el mundo justo y el sexismo, sino plantear estrategias para combatirlo de manera eficaz.

Respecto a las limitaciones del presente estudio, se identifica que la mayor parte de la muestra se conformó por personas con escolaridad licenciatura y jóvenes, características que no son representativas de la totalidad de la población mexicana. Las características de esta muestra pueden implicar un sesgo respecto a los resultados, ya que, al ser una muestra compuesta por jóvenes, implicaría que se encuentran en un periodo de vida en el que cuestionan el statu quo y, por lo tanto, las tendencias culturales machistas. También debido al nivel de estudios que tienen, es probable que tiendan a debatir sobre las creencias y comportamientos sexistas que otro tipo de muestra no cuestiona. Por ello, para futuras investigaciones será necesario realizar estudios en muestras con características más heterogéneas, dado que gran parte de la población mexicana tiene un menor nivel de estudios.

También, para futuras investigaciones será necesario plantear modelos explicativos que incluyan más variables para predecir el sexismo ambivalente en hombres y mujeres, por ejemplo, la crianza, las premisas culturales y los rasgos de personalidad, por lo que es necesario explorarlas e incluir muestras con características sociodemográficas más diversas, con la 
finalidad de generar un modelo que permita conocer a fondo las variables que explican el fenómeno.

\section{Conclusiones}

La creencia en el mundo justo tiene una función adaptativa y paliativa, ya que es utilizada como una estrategia para enfrentarse a las condiciones de la sociedad desigual, sin embargo, también permite justificar las posiciones de poder de los grupos de alto estatus.

Esta creencia se relaciona con el sexismo benevolente y el sexismo hostil tanto para hombres como para mujeres, ya que ambos grupos forman parte de un sistema en el que se legitima la inequidad y el orden social desigual. Por ello, es necesario generar estrategias adaptativas que permitan enfrentarse al sistema social, pero que tengan un enfoque hacia la igualdad y que permitan reducir el prejuicio hacia las mujeres.

\section{Agradecimientos}

Se agradece al Consejo Nacional de Ciencia y Tecnología (CONACYT) por la beca otorgada a la primera autora del presente artículo, con número de becario 581389, CVU 572614 y número de beca 412829 , con la que se financió la presente investigación.

\section{Referencias}

Acuña, M. E., \& Montecino, S. (2014). La otra reforma: El no sexismo como clave cultural del cambio en el sistema educacional. Revista Anales, 7(1), 109-120.

Arenas-Rojas, A., \& Rojas-Solís, J. (2015). Sexismo ambivalente hacia hombres: Un estudio exploratorio con adolescentes mexicanos. REIDOCREA, 4(8), 54-59.

Arnoso, A., Babe, I., Arnoso, M., \& Elgorriaga, E. (2017). El sexismo como predictor de la violencia de pareja en un contexto multicultural. Anuario de Psicología Jurídica, 27(1), 9-20. https://doi.org/10.1016/j.apj.2017.0 2.001

Blanton, H., George, G., \& Crocker, J. (2001). Contexts of system justification and system evaluation: Exploring the social comparison strategies of the (not yet) contented female worker. Group Processes \& Intergroup Relations, 4(2), 126-137. https://doi.org/10.1177/1368430201004002 004

Brown, R. (2010). Prejudice: Its social psychology. Malden, MA, Estados Unidos: Wiley-Blackwell.

Cárdenas, M., Lay, S., González, C., Calderón, C., \& Alegría, I. (2010). Ambivalent sexism inventory: Adaptation, validation and relationship to psychosocial variables. Salud \& Sociedad, 1(2), 125-135.

Carrera, R. (2018). El uso de metáforas en la construcción del discurso sobre el sexismo: La situación de México (Tesis de maestría). Norges Arktiske Universitet, Noruega. 
Castillo, A., \& Maldonado, M. (1996). Evitación del éxito en mujeres amas de casa y sus correlatos (Tesis de liciendado). Universidad Nacional Autónoma de México, México.

Chen, Z., Fiske, S., \& Lee, T. (2009). Ambivalent sexism and power-related gender-role ideology in marriage. Sex Roles, 60(11-12), 765-778. https:/ /doi.org/10.1007/s11199-009-9585-9

Cikara, M., Lee, T., Fiske, S., \& Glick, P. (2009). Ambivalent sexism at home and at work: How attitudes toward women in relationships foster exclusion in the public sphere. En J. Jost, A. Kay, \& H. Thorisdottir (Eds.), Social and psychological bases of ideology and system justification (pp. 444-462). Nueva York, NY, Estados Unidos: Oxford University Press.

Crocker, J., \& Quimm, D. (2001). Psychological consequences of devalued identities. En R. Brown \& S. Gaertne (Eds.), Handbook of social psychology: Intergroup processes (pp. 238-257). Oxford, Reino Unido: Blackwell Publishers.

Crosby, F., Stockdale, M., \& Ropp, A. (1947). Sex discrimination in the workplace. Oxford, Reino Unido: Blackwell Publishers.

Cruz, C. (2013). Modelo explicativo del sabotaje y violencia hacia miembros del propio grupo en grupos de bajo estatus (Tesis de doctorado). Universidad Nacional Autónoma de México, México.

Cruz, C., Zempoaltecatl, V., \& Correa, F. (2005). Perfiles de sexismo en la Ciudad de México: Validación del Cuestionario de Medición del Sexismo Ambivalente. Enseñanza e Investigación en Psicología, 10(2), 381-395.

Cusick, T. (1987). Sexism and early parenting: Cause and effect? Peabody Journal of Education, 64(4), 113-131. https://doi.org/10.1080/0161956 8709538573

Díaz-Guerrero, R. (1994). Psicología del mexicano: Descubrimiento de la etnopsicología. CDMX, México: Trillas.

Díaz-Loving, R., González-Rivera, I., \& Baeza-Rivera, M. J. (2019). Sexismo: Una configuración a partir de las premisas histórico-psicosocioculturales. Enseñanza e Investigación en Psicología, 1(3), 287-293.

Díaz-Loving, R., Velasco-Matus, P., \& Rivera-Aragón, S. (2018). Felicidad, instrumentalidad y expresividad en dos muestras latinoamericanas: México y Bolivia. Persona, 21(1), 11-29.

Díaz-Loving, R, Rivera, S., \& Sanchez, R. (2001). Rasgos instrumentales (masculinos) y expresivos (femeninos), normativos (típicos e ideales) en México. Revista Latinoamericana de Psicología, 33(2), 131-139.

Díaz-Loving, R., Saldívar, A., Armenta-Hurtarte, C., Reyes, N. E., López, F., Moreno, M., ... Correa, F. E. (2015). Creencias y normas en México: Una actualización del estudio de las premisas psico-socio-culturales. Psykhe, 24(2), 1-25. https://doi.org/10.7764/psykhe.24.2.880

Durante, F., Fiske, S. T., Kervyn, N., Cuddy, A. J. C., Akande, A. D., Adetoun, B. E., ... Storari, C. C. (2013). Nations' income inequality predicts ambivalence in stereotype content: How societies mind the gap. British Journal of Social Psychology, 52(4), 726-746. https://doi.org/10.1111/bj so. 12005

Eagly, A., \& Carli, L. (2003). The female leadership advantage: An evaluation of the evidence. Leadership Quarterly, 14(6), 807-834. https://doi.org/10.1 016/j.leaqua.2003.09.004 
Ellemers, N., \& Barreto, M. (2001). The impact of relative group status: Affective, perceptual and behavioral consequences. En R. Brown \& S. Gaertner (Eds.), Handbook of social psychology: Intergroup processes (pp. 324-343). Oxford, Reino Unido: Blackwell Publishers. https://doi.org/1 0.1002/9780470693421.ch16

Fernández, M. (2018). El embarazo adolescente como dispositivo de sexualidad en la formación de roles de género (Tesis de pregrado). Universidad de Chile, Chile.

Gatica, L., Martini, J., Dreizik, M., \& Imhoff, D. (2017). Predictores psicosociales y psicopolíticos de la justificación de la desigualdad social. Revista de Psicologia, 35(1), 279-310. http://doi.org/10.18800/psico.20 1701.010

Glick, P., \& Fiske, S. (1996). The Ambivalent Sexism Inventory: Differentiating hostile and benevolent sexism. Journal of Personality and Social Psychology, 70(3), 491-512. https://doi.org/10.1037/0022-3514.70.3.491

Glick, P., Fiske, S., Mladinic, A., Saiz, J., Abrams, D., Masser, B., \& López, W. (2000). Beyond prejudice as simple antipathy: Hostile and benevolent sexism across cultures. Journal of Personality and Social Psychology, 79(5), 763-775. https://doi.org/10.1037/0022-3514.79.5.763

Gonzalez-Rivera, I., \& Díaz-Loving, R. (2018). Predictores del sexismo ambivalente hacia los hombres. Acta de Investigación Psicológica, 8(3), 4352. http://dx.doi.org/10.22201/fpsi.20074719e.2018.3.05

Hafer, C., \& Choma, B. (2009). Belief in a just world, perceived fairness, and justification of the status quo. En J. Jost, A. Kay \& H. Thorisdottir (Eds.), Social and psychologica bases of ideology and system justification (pp. 107125). Oxford, Reino Unido: Oxford University Press.

Hafer, C., \& Olson, J. (1993). Beliefs in a just world, discontent, and assertive actions by working women. Personality and Social Psychology Bulletin, 19(1), 30-38. https://doi.org/10.1177\%2F0146167293191004

Jost, J. (1997). An experimental replication of the depressed-entitlement effect among women. Psychology of Women Quarterly, 21(3), 387-393. https:// doi.org/10.1111/j.1471-6402.1997.tb00120.x

Jost, J., \& Banaji, M. (1994). The role of stereotyping in system-justification and the production of false consciousness. British Journal of Social Psychology, 33(1), 1-27. https://doi.org/10.1111/j.2044-8309.1994.tb01008.x

Jost, J., \& Hunyady, O. (2002). The psychology of system justification and the palliative function of ideology. European Review of Social Psychology, 13(1), 111-153. https://doi.org/10.1080/10463280240000046

Jost, J., \& Hunyady, O. (2005). Antecedents and consequences of systemjustifying ideologies. Current Directions in Psychological Science, 14(5), 260-265. https://doi.org/10.1111/j.0963-7214.2005.00377.x

Kaiser, C., Hagiwara, N., Malahy, L., \& Wilkins, C. (2009). Group identification moderates attitudes toward ingroup members who confront discrimination. Journal of Experimental Social Psychology, 45(4), 770777. https://doi.org/10.1016/j.jesp.2009.04.027

Kay, A., \& Jost, J. (2003). Complementary justice: Effects of "poor but happy" and "poor but honest" stereotype exemplars on system justification and implicit activation of the justice motive. Journal of Personality and Social Psychology, 85(5), 823-837. https://doi.org/10.1037/0022-3514.85.5.82 3 
Kay, A., \& Zanna, M. (2009). A contextual analysis of the system justification motive and its societal consequences. En J. Jost, A. Kay \& H. Thorisdottir (Eds.), Social and psychological bases of ideology and system justification (pp. 158-181). Oxford, Reino Unido: Oxford University Press. https://doi.or g/10.1093/acprof:oso/9780195320916.003.007

Leite, A., Cardoso, S., Marques, A., \& Morais, C. (2017). 'Creer es adaptarse': Creer en un mundo justo y la identificación y satisfacción de los emigrantes con el país de acogida. Revista de Psicología Social, 32(2), 424-439. https: //doi.org/10.1080/02134748.2017.1291743

Luna, D. (2008). Una aproximación al estudio del sexismo ambivalente en México (Tesis de pregrado). Universidad Nacional Autónoma de México, México.

Luna, D. (2010). Análisis de datos psicometricos con dos modelos de variables latentes (Tesis de maestría). Universidad Nacional Autónoma de México, México.

Molix, L. (2014). Sex differences in cardiovascular health: Does sexism influence women's health? The American Journal of the Medical Sciences, 348(2), 153-155. https://doi.org/10.1097/MAJ.0000000000000300

Moya, M., Vasco, P., Glick, P., \& Poeschl, G. (2002). Masculinidad y feminidad y factores culturales. Revista Española de Motivación y Emoción, 3, 127-142.

Mummendey, A., \& Otten, S. (2001). Aversive discrimination. En R. Brown \& S. Gaertner (Eds.), Handbook of social psychology: Intergroup processes (pp. 112-132). Oxford, Reino Unido: Blackwell Publishers.

O'Brien, L., \& Major, B. (2009). Group status and feelings of personal entitlement: The roles of social comparison and system-justifying beliefs. En J. Jost, A. Kay \& H. Thorisdottir (Eds.), Social and psychologica bases of ideology and system justification (pp. 427 - 443). Oxford, Reino Unido: Oxford University Press.

Pelham, B. W., \& Hetts, J. J. (2001). Underworked and overpaid: Elevated entitlement in men's self-pay. Journal of Experimental Social Psychology, 37(2), 93-103. https://doi.org/10.1006/jesp.2000.1429

Phinney, J. (1990). Ethnic identity in adolescents and adults: Review of research. Psychological Bulletin, 108(3), 499-514. https://doi.org/10.1037/00332909.108.3.499

Reyes Lagunes, I., \& García y Barragán, L. F. (2008). Procedimiento de validación psicométrica culturalmente relevante: Un ejemplo. $L a$ Psicología Social en México, XII(1), 625-630.

Rocha, S. T., \& Díaz-Loving, R. (2004). Réplica y extensión de la escala de rasgos de instrumentalidad y expresividad. Psicología Social de México, 10(1),7986.

Rojas, P., \& Moreno, R. (2016). Sexismo hostil y benevolente en adolescentes. Una aproximación ético-cultural. Revista Iberoamericana de Educación, 72(1), 31-46.

Rudman, L., \& Glick, P. (2001). Prescriptive gender stereotypes and backlash toward agentic women. The Journal of Social Issues, 57(4), 743-762. http s://doi.org/10.1111/0022-4537.00239

Uhlmann, E., Poehlman, A., \& Bargh, J. (2009). American moral exceptionalism. En J. Jost, A. Kay \& H. Thorisdottir (Eds.), Social and psychologica bases of ideology and system justification (pp. 27 - 52). Oxford, Reino Unido: Oxford University Press. 
Verdú, A., \& Briones, E. (2016). Desigualdad simbólica y comunicación: El sexismo como elemento integrado en la cultura. Revista de Estudios de Género La Ventana, 44(1), 24-50. 